\title{
Special Issue on Future Digital Design and Manufacturing: Embracing Industry 4.0 and Beyond-Part II
}

\author{
Sheng-Feng Qin ${ }^{1}$ Kai Cheng ${ }^{2}$
}

Published online: 1 August 2017

(C) Chinese Mechanical Engineering Society and Springer-Verlag GmbH Germany 2017

Digital design and manufacturing have been under pinned by digital modeling, simulation, and automation controls for decades. Under the new market requirement of mass customized products and services, the advancements in artificial intelligence (AI), smart technology, virtual reality (VR), big data, digital twin, robotics and human-centered design are becoming driving forces for the development of future digital design and manufacturing. This special issue focuses on the future digital design and manufacturing especially under the Industry 4.0 framework and beyond. This editorial introduces the papers in this special issue, which linked to the International Workshop on Digital Design and Manufacturing Technologies - Embracing Industry 4.0 and Beyond at Northumbria University in Newcastle, UK, held on 12-13 April 2016. In the Part I of the issue [1], there are 13 papers published in 2016, Volume 29, No 6 of the Chinese Journal of Mechanical Engineering (this journal).

In the Part II, there are 16 papers selected for publication, covering several aspects such as design and manufacturing complexity, integrated multidisciplinary design, robotics and smart product/system design, smart tooling design, and smart manufacturing and production. The thematic links of all selected papers to the future design and manufacturing for supporting Industry 4.0 and beyond are discussed in the complementary article- the Research Highlight in this issue.

Sheng-Feng Qin

sheng-feng.qin@northumbria.ac.uk

1 School of Design, Northumbria University, Newcastle upon Tyne NE1 8ST, UK

2 College of Engineering, Design and Physical Sciences, Brunel University London, Uxbridge UB8 3PH, UK
First, there is a need of an applicable framework for Industry 4.0 to guide future digital design and manufacturing research. Professor Muller and Dr Chen in their invited keynote paper revealed the shortcomings of existing researches as the lacking of an applicable framework, and then provided a reference architecture with four perspectives in terms of the manufacturing process, devices, software and engineering. In addition, they provided a special insight with the usage of Cyber-Physical Systems (CPS), accompanied with case studies.

In the research about the complex product development and integrated multidisciplinary design, $\mathrm{M}$ Aftab and $\mathrm{A} \mathrm{H}$ Rusli investigated the role of digitization of products and ways it affect emotional design of visceral, behavioral and reflective products. The research concluded that digitization could give products the ability to capture, store and then communicate the stories, journey and memories back, in order to empower people to understand the value of longer-term use of products. It is important to understand the user/customer's need/want/expect in human centered design and how to transfer customers' requirements into design specifications is equally important in design practice. X J Ma and his colleagues studied ways of transforming multidisciplinary customer requirements to product design specifications, which is a very important starting point for new product development. Furthermore, when changes are made in a product design specification, how to effectively find key change propagation paths is essentially important for rapid and intelligent design task planning. $\mathrm{H} \mathrm{Z}$ Zhang et al. investigated design change modelling with this regard. On the other hand, S N Xiao and co-authors investigated a complex network analysis approach to clarifying the relationships between product design parameters, boundary conditions and performance index in engineering design and establishing the top-down 
design of a product. Q C Sun, et al. studied a design structure matrix (DSM) based method to determine task relationships in correlated multiple design projects and help design information flow scheduling for supporting concurrent multi-product development. G Z Hu with his colleagues proposed a product data model for performancedriven design.

As for the robotics and smart product/system, J Z Fan, et al., investigated a mimicry-based design and dynamic modelling method for designing a frog-inspired swimming robot powered by pneumatic muscles. While S B Jung and the co-authors explored challenges and opportunities in developing a smart and effective driving assistive system for the elderly drivers, with regarding to the autonomous vehicles. The interactions between humans (users) and machines/systems are also a point of study. K Ding and P Y Jiang proposed a hardware and software integrated cloud platform (or mediator) for social manufacturing systems under mass individualization environment, which has a potential of facilitating multi-level production interactions, such as human-to-human, human-to-machine and machineto-machine interactions.

In the field of smart tooling, smart manufacturing and production, $\mathrm{K}$ Cheng and his colleagues, discussed and demonstrated the development, implementation and application perspectives of smart machining with smart cutting tools. In smart manufacturing, for improving production efficiency and reducing labor costs, $\mathrm{C} \mathrm{Z}$ Guo, et al. conducted an experimental dynamic analysis of a breathing cracked rotor, aiming to identify feasible features for the self-aware health management of rotating machinery through fault diagnostics and prognostics. G H Song, et al. investigated design optimization of irregular cellular structures for additive manufacturing. In smart production, J Q Zhang and co-authors, demonstrated a new cylinder movement simulation model based on aerodynamics in order to accurately calculate the takt of production line. X P Xiao, et al. presented a new method for measured surface localization for machining quality inspection and error compensation of machining tools. While M K Zheng with colleagues proposed an integrated approach of
Bayesian network and big data analytics for quality analysis and control of manufacturing processes.

We are fully aware of the on-going research and development efforts and the associated discussion on Industry 4.0 and beyond within the manufacturing research community and the industry. It hopes the selected papers in the special issue can provide some in-sight for future research and development, and good references for industrial practices.

\section{Reference}

1. Kai Chen. Special Issue on Future Digital Design and Manufacturing: Embracing Industry 4.0 and Beyond. Chinese Journal of Mechanical Engineering, 2016, 29(6): 1045. DOI: 10.3901/CJME. 2016.0909.110.

Sheng-Feng Qin is a Full Professor of Digital Design, Northumbria University Newcastle, UK. He received his $\mathrm{PhD}$ degree in Design Technology from University of Wales, UK, in 2000. Before joining Northumbria School of Design, he was a Lecturer and then Senior Lecturer in Product Design at Brunel University between 2002 and 2013. Before that, He was a post-doctoral Research Fellow at Loughborough University between 2000 and 2001, and a Research Assistant at Cardiff Metropolitan University between 1998 and 1999. From 1996 to 1997, he was an Academic Visiting Scholar at Birmingham University and an Assistant/Associate Professor at East China Jiaotong University of China between 1988 and 1996. His research interest include digital design and manufacturing. He has authored/coauthored over 160 papers.

Kai Cheng is a chair Professor in Manufacturing Systems and currently the Theme Leader for Ultraprecision and Micro/Nano Manufacturing Theme at Brunel University London. His research interests include ultraprecision machining, design of high precision machines, smart tooling and smart machining, and sustainable manufacturing systems. Professor Cheng and his team have enjoyed working closely with industrial companies in the UK, Europe, USA and Far East. Professor Cheng is a Charted Engineer and a Fellow of the IMechE and IET, the European Editor for International Journal of Advanced Manufacturing Technology and a member of the Editorial Board at other 4 international journals. Professor Cheng is also honored with the Qianren Professorship at Harbin Institute of Technology, China. 